Proc. 13th International School on Theoretical Physics: Symmetry and Structural Properties of Condensed Matter

\title{
A Statistical Method for Calculating the Velocity of Acoustic Waves in Extreme Conditions
}

\author{
H. CZYŻ $\dot{Z}^{a *}$, T. JASIŃSKI ${ }^{a}$ AND A. WŁOCH ${ }^{b}$ \\ ${ }^{a}$ Department of Physics and Medical Engineering, Faculty of Mathematics and Applied Physics, \\ Rzeszów University of Technology, al. Powstańców Warszawy 12, 35-959 Rzeszów, Poland \\ ${ }^{b}$ Department of Mathematical Modeling, Faculty of Mathematics and Applied Physics, \\ Rzeszów University of Technology, al. Powstańców Warszawy 12, 35-959 Rzeszów, Poland
}

\begin{abstract}
In this paper the sound wave velocity in gases has been computed. Consideration has been given to the statistical dependence of specific heat on the temperature. Thus instead of the classical formula where the thermodynamic adiabatic exponent $\kappa$ is a constant quantity, statistical expressions have been obtained. Numerical results as well as a graph which presents the difference of results obtained by two methods are given and analytical results are obtained and compared.
\end{abstract}

DOI: 10.12693/APhysPolA.135.1231

PACS/topics: velocity of acoustic wave, statistical method, high temperature, thermodynamic adiabatic exponent

\section{Introduction}

The work presented here concerns the issue of the propagation of acoustic waves in gases at high temperatures. The subject contains important aspects for modern aviation and rocket technology [1]. The formula expressing acoustic wave velocity depends on the thermodynamic adiabatic exponent $\kappa$, it is constant on low temperature and is determined by the structure of the gas molecules. In extreme conditions, e.g. high temperature, vibrations of the molecules play an important role. In these cases the dependence of the acoustic wave velocity on the temperature is complicated. For molecular acoustics it is important to determine the theoretical velocity of acoustic waves $c$ as a function of temperature $T$. The equations of state can directly determine the dependence of $c(T)$ for various models of gases, with the assumption that the gas is well above critical state. In an ideal gas particles have the linear and rotational kinetic energy, oscillation energy, and the excitation energies of the electrons. Each one of types of motion is activated after its characteristic temperature is reached.

According to the principles of statistical physics the specific heat $c_{p}$ and $c_{\nu}$ for gases depend on temperature and the adiabatic equation is not applicable in the Clapeyron equation. The adiabatic equation can then be written only in the integral form [2]. Translational motion is not quantized and the allowed energy levels form a continuum. Rotational motion is quantized and the distance between the energy levels depends on the moment of inertia of the particles. Oscillatory motion is quantized, the distance between the energy levels depends on

*corresponding author; e-mail: hczyz@prz.edu.pl the masses of the atoms forming the molecule, and the binding energy between them. Electron energy levels are quantized [3].

Models of particles can be described by their degrees of freedom $f$. This is the smallest number of generalized coordinates required to describe the motion of the molecule. If $n$ is the number of atoms in the molecule, then the number of degrees of freedom is $f=3 n$. For a single atom molecule these are the three translational degrees of freedom. Polyatomic molecules have three translational degrees of freedom, and two or three rotational degrees of freedom, depending on whether the molecule is linear or nonlinear. For linear molecules the number of vibrational degrees of freedom is equal to $3 n-5$. For nonlinear molecules the number of vibrational degrees of freedom is equal to $3 n-6$.

Electronic excitations of molecules are present only at temperatures of $10^{4} \mathrm{~K}$ and will not be considered in this paper. The occurrence of rotational excitations depends on the ratio: $\frac{\theta_{\text {rot }}}{T}$ where $\theta_{\text {rot }}$ is the characteristic temperature of rotation for a given molecule.

At room temperatures, it is assumed that every degree of freedom of the molecules per mole of gas accounts for heat equal to half the value of the universal gas constant $R$. Then the value of $\kappa$ depends only on the number of degrees of freedom $f: \kappa=\frac{f+2}{f}$. At higher temperatures the specific heat cannot be captured using the degrees of freedom [4].

\section{Description of a statistical method for calculating the velocity of acoustic waves}

According to the principles of statistical physics the specific heat $c_{p}$ and $c_{\nu}$ for gases depend on temperature. The adiabatic equation can then be written as the integral [2]: 


$$
\ln \rho=\frac{1}{R} \int c_{\nu}(T) \frac{\mathrm{d} T}{T}+\text { const, }
$$

where $\rho$ is the density of the gas and $R$ is the universal gas constant.

Writing the first law of thermodynamics in the form containing the adiabatic enthalpy equation we get in the integral

$$
\ln p=\frac{1}{R} \int c_{p}(T) \frac{\mathrm{d} T}{T}+\text { const },
$$

where $p$ is the pressure of the gas.

The adiabatic acoustic wave velocity is the derivative of the density of the pressure at constant enthalpy

$$
c_{0}^{2}=\left(\frac{\mathrm{d} p}{\mathrm{~d} \rho}\right)_{s}=\frac{c_{p}(T)}{c_{\nu}(T)} \frac{p}{\rho}=\kappa(T) \frac{p}{\rho} .
$$

Hence we obtain

$$
c_{0}=\sqrt{\kappa(T) \frac{R}{\mu} T} .
$$

The calculation uses the classical statistics of MaxwellBoltzmann [3], counting the quantum energy of gas molecules. Finally,

$$
c_{0}=\sqrt{\left(1+\frac{R}{c_{\nu}(T)}\right) \frac{R}{\mu} T}
$$

where $\mu$ is the molecular mass.

For diatomic gases the statistical sum of the energies of the states is affected by translational, rotational and vibrational motion. In the case of hydrogen one must also consider the influence of electron levels. The contribution of the internal degrees of freedom to the partition function $Z$ is as follows [5]:

$$
Z=Z_{t r} Z_{o s c} Z_{\text {rot }},
$$

where partition function $Z_{t r}$ is the translational, $Z_{o s c}$ is the oscillatory function and $Z_{\text {rot }}$ is the rotational.

The translational partition is as follows:

$$
Z_{t r}=\sum_{i} \exp \left(-\frac{\mu \nu_{i}^{2}}{2 k T}\right)=\frac{V}{h^{3}}(2 \pi \mu k T)^{\frac{3}{2}},
$$

where $V$ is the volume of the area and $k$ is the Boltzmann constant.

The oscillatory partition function is calculated assuming that the atoms in a diatomic molecule are harmonic oscillators

$$
Z_{o s c}=\sum_{n=0}^{\infty} \exp \left(-\frac{\left(n+\frac{1}{2}\right) h \nu}{k T}\right),
$$

where $h$ is Planck's constant and $\nu$ is the frequency denoting the characteristic temperature oscillations,

$$
\theta_{\text {osc }}=\frac{h \nu}{k} \text {. }
$$

Summing over the geometric progression we find

$$
Z_{o s c}=\frac{\exp \left(-\frac{\theta_{o s c}}{2 T}\right)}{1-\exp \left(-\frac{\theta_{o s c}}{T}\right)} .
$$

The rotational distribution function is as follows [2]:

$$
Z_{\text {rot }}=\sum_{n=0,2,4, \ldots}(2 n+1) \exp \left(-(n+1) n \frac{\theta_{\text {rot }}}{T},\right)
$$

where the characteristic temperature of rotation is

$$
\theta_{\text {rot }}=\frac{\hbar^{2}}{2 I k}
$$

with $I$ the moment of inertia of the molecule.

Rotational energy levels are $(2 n+1)$ times degenerate. At high temperatures the summation of the discrete values of $n$ can be replaced by integration over the continuous values of $n$, resulting in

$$
Z_{\text {rot }}=\frac{\theta_{\text {rot }}}{T} \text {. }
$$

$Z$ for the diatomic molecule is given by

$$
Z=\frac{V}{h^{3}}(2 \pi m k T)^{\frac{3}{2}} \frac{\exp \left(-\frac{\theta_{o s c}}{2 T}\right)}{1-\exp \left(-\frac{\theta_{o s c}}{T}\right)} \frac{T}{\theta_{\text {rot }}} .
$$

Knowing the distribution function $Z$ we can calculate the internal energy per mole $U$ :

$$
U=R T^{2}\left(\frac{\partial \ln Z}{\partial T}\right)_{\nu} .
$$

The internal energy per mole is the sum of the internal energies translation $U_{t r}$, oscillation $U_{o s c}$, and rotation $U_{\text {rot }}$ :

$$
U=U_{t r}+U_{o s c}+U_{\text {rot }} .
$$

The specific heat of a gas at a constant volume

$$
c_{\nu}=\left(\frac{\partial U}{\partial T}\right)_{\nu} \text {. }
$$

Finally, one obtains

$$
c_{\nu}=R\left\{\frac{3}{2}+\left(\frac{\theta_{o s c}}{T}\right)^{2} \frac{\exp \left(\frac{\theta_{o s c}}{T}\right)}{\left[\exp \left(\frac{\theta_{o s c}}{T}\right)-1\right]^{2}}+1\right\} .
$$

Substituting (18) into (4) we obtain the expression for the statistical adiabatic acoustic wave velocity in a gas of diatomic molecules

$$
c_{0}^{2}=\frac{R T}{\mu}\left[1+\frac{1}{\frac{5}{2}+\frac{\xi^{2} \exp \xi}{(\exp \xi-1)^{2}}}\right],
$$

where $\xi=\frac{\theta_{o s c}}{T}$.

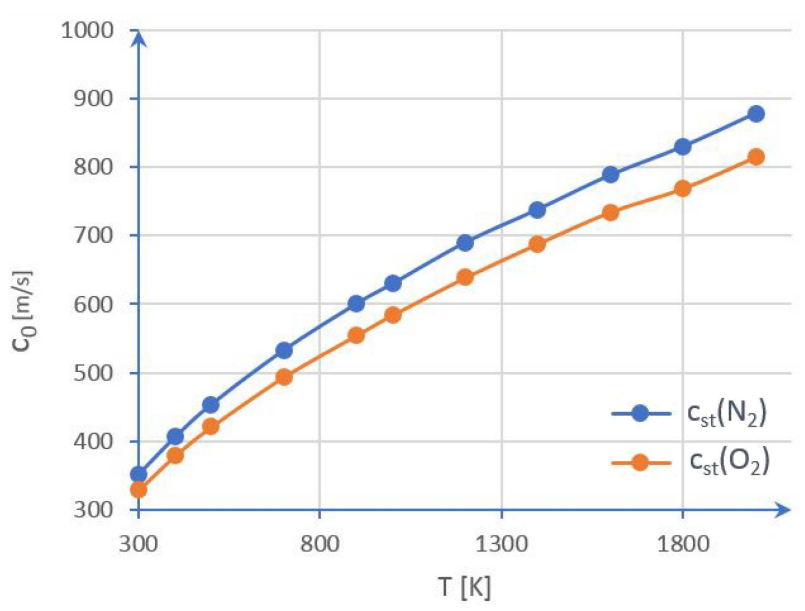

Fig. 1. The acoustic waves velocity versus temperature for nitrogen and oxygen obtained by statistical methods. 


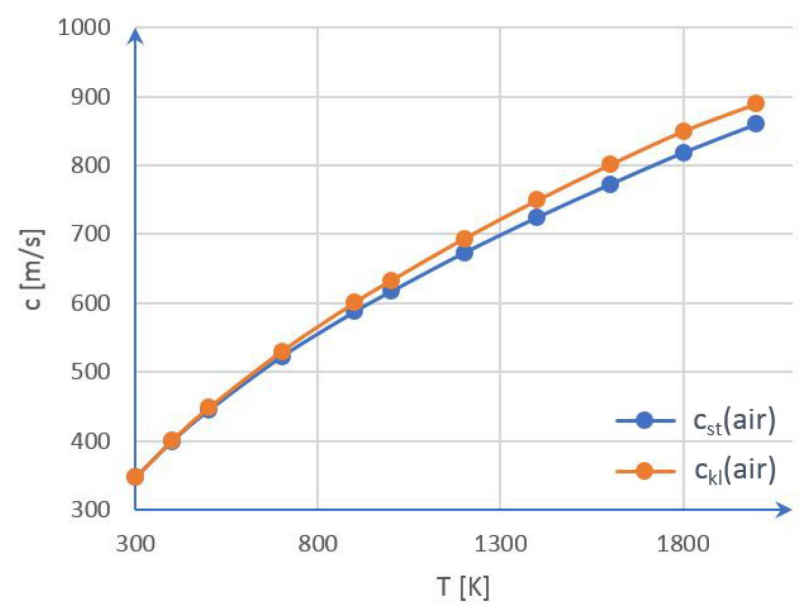

Fig. 2. The acoustic waves velocity versus temperature for air, where $c_{k l}$ is obtained by "classical" methods [6] and $c_{s t}$ is obtained by statistical methods.

Detailed calculations have been made for air (a mixture of nitrogen and oxygen). The results of calculations are presented in Figs. 1 and 2.

\section{Conclusions}

Summarizing the considerations presented here we would like to first emphasize that the present issue has important practical implications, especially in aviation technology [7, 8]. On the basis of detailed calculations of the acoustic wave velocity in the air it was concluded that even starting from a temperature of $300 \mathrm{~K}$ there is a significant difference between the value rate calculated by the formula: $c_{0}=\sqrt{\kappa \frac{R}{\mu} T}$ and by formula (5). This difference increases with increasing temperature and at a temperature $2000 \mathrm{~K}$ is of the order of several percent. At a temperature of $2000 \mathrm{~K}$ the degree of dissociation of oxygen and nitrogen molecules is very small. It can therefore be regarded as a diatomic gas.

However, if a large percentage of the gas molecules are dissociated, the reported models no longer apply.

\section{References}

[1] B. Etkin, Dynamics of Atmospheric Flight, Wiley, New York 2005.

[2] S.R. Berry, S.A. Rice, J. Ross, Matter in Equilibrium: Statistical Mechanics and Thermodynamics, Oxford University Press, 2001.

[3] H. Gould, J. Tobochnik, Statistical and Thermal Physics, Princeton University Press, 2010.

[4] J. D. Anderson, Hypersonic and High Temperature Gas Dynamics, American Institute of Aeronautics and Astronautics, 2000.

[5] H. Czyż, "Propagation of acoustic wave in polyatomic gases in high temperatures", in: Proc. 59th Open Seminar of Acoustics, 2012, Ed. A. Jozefczak, Polish Acoustical Society, Poznań 2012.

[6] H. Czyż, Dispersed phase acoustics in: Selected Problems of Theory of Acoustic Field, FOSZE, Rzeszów 1994 (in Polish).

[7] E.H. Herschel, Basics Of Aerothermodynamics, Vol. 206, Springer, 2005.

[8] U. Walter, Astronautics: The Physics of Space Flight, Wiley, 2012. 